\title{
Designing Games for Healthy Sleep
}

\author{
Juliet Pusateri, Judith Leng, Qian Wang, Xiangzhu Chen, Jessica Hammer \\ Carnegie Mellon University \\ Pittsburgh PA, USA \\ jpusater@alumni.cmu.edu, judithl@alumni.cmu.edu,wangqian.evelyn@gmail.com, cxzeva@gmail.com, \\ hammerj@andrew.cmu.edu
}

\begin{abstract}
A sleep deficit has far-reaching consequences, but for many people, healthy sleep is not a priority or a possibility. We explore the potential for "sleepy games" as a genre of transformational games. To explore this design space, we prototyped nine games through an iterative design process. Based on analysis of design decisions and the games as artifacts, we identify seven design challenges for sleepy games: agency and control; physiological and mental arousal; intervention timing; social embeddedness; multisensory experience; vulnerability; and identity and values. We expand on three games with playtesting to show how these design challenges unfold for players in practice, show the impact on players' lives, and discuss sleepy games as creative, social, and situated practices.
\end{abstract}

\section{Author Keywords}

Game design; transformational games; sleep; games for health.

\section{CCS Concepts \\ -Human-centered computing $\rightarrow$ Human computer inter- action (HCI);}

\section{INTRODUCTION}

A key factor influencing quality of life is getting a good night of sleep. Lack of sleep affects a range of outcomes including learning [55]; risk for Alzheimer's, heart disease, and cancer [54]; and mortality [22]. Healthy sleep enables us to think, play, work, and function effectively. Yet, more than 30 percent of adults [20] and 70 percent of adolescents [18] are sleep deprived in the United States. Contemporary culture pushes for ever-increasing pace and productivity. For example, the ubiquity of smartphones means they are always within arm's reach, while workers like nurses or truck drivers may be required to sleep in ways that out of sync with the Circadian rhythms of the body. These factors result in people pushing the limits on both the quantity and quality of their sleep.

Permission to make digital or hard copies of all or part of this work for personal or classroom use is granted without fee provided that copies are not made or distributed for profit or commercial advantage and that copies bear this notice and the full citation on the first page. Copyrights for components of this work owned by others than ACM must be honored. Abstracting with credit is permitted. To copy otherwise, or republish, to post on servers or to redistribute to lists, requires prior specific permission and/or a fee. Request permissions from permissions@acm.org.

CHI '20, April 25-30, 2020, Honolulu, HI, USA.

(C) 2020 Association for Computing Machinery.

ACM ISBN 978-1-4503-6708-0/20/04 \$15.00.

http://dx.doi.org/10.1145/3313831.3376413
What is the role of technology in supporting healthy sleep? Existing efforts focus on educating users, visualizing users' sleep data, helping with sleep timing, and encouraging reflection. Games can offer further ways to help with sleep, such as changing behavior with embedded design techniques [36], but work to date on sleep and games has been limited. As of now, little is known about how to create effective "sleepy games," or games that support healthy sleep.

To begin to fill this gap, we adopt a research through design approach [57]. Nine different game design teams developed playable prototypes over the course of eight months, in response to sleep problems identified by health experts. Each prototype received at least five rounds of internal playtesting and iteration, with detailed feedback from playtesters, game design experts, sleep experts, and other members of the research team. Designers produced and discussed a range of artifacts; these design decisions led to the identification of seven design challenges for sleepy games [47].

In this paper, we deepen our understanding of these design challenges: agency and control; physiological and mental arousal; intervention timing; social embeddedness; multisensory experience; vulnerability; and identity and values. We do so through the lens of three games from our design set, selected for coverage of all seven challenges. We conducted 12 field tests of each game with external participants. We then incorporate insights from our design discussions and findings from these playtests to unpack our challenges. While our field tests were not designed to measure behavior change outcomes, we nonetheless observed that the games changed players' relationship to sleep by providing opportunities for experimentation, reflection, and conversation.

Finally, we show how understanding sleep as a creative, social, and situated practice can lead to new opportunities for game design. These include: the opportunity for artifacts to redirect player agency around sleep activities; the opportunity to design games for people adjacent to a sleep problem; and the opportunity to use choices made during gameplay to affect the physical or social context of sleep.

\section{LITERATURE REVIEW}

\section{Obstacles to Healthy Sleep}

Adequate sleep for adults refers to a minimum of seven hours of restorative rest everyday; for children, it's over ten [29]. Obtaining and maintaining healthy sleep is not as easy as it 
might sound; there are several types of barriers that prevent needed sleep.

First, sleep disorders including insomnia, sleep apnea, restless leg syndrome, and narcolepsy may impede healthy sleep. Sleep disorders are tied to genetic, psychological, and medical factors [4]. Other physical or psychological conditions can also affect sleep, such as chronic pain or anxiety[4].

Second, a person's environment may not be conducive to sleep. For example, they may have to sleep in a place that is noisy or brightly lit [28] [45] [53]. Their sleep environment may include regular interruptions, such as nurses or doctors who are on-call during the night [21]. Sleepers also may not have access to the resources that would allow them to sleep well, such as going to bed hungry because no food is available.

Third, people may have trouble establishing a regular sleepwake cycle. For example, shift workers can have difficulty following a regular work-sleep schedule if working nighttime hours. If a shift worker's schedule is out of sync with their biological clock, they may be required to work while their body is telling them to sleep, and vice versa [21]. In an attempt to make up for missing sleep, some shift workers try to "bank" their sleep; however, even a single night of sleep deprivation can affect memory and cognition [27]. Sleep-wake cycles can also be disrupted by periodic, rather than chronic, events. For example, jet lag disrupts a regular sleep cycle for people whose jobs require frequent travel [52].

Fourth, social norms around work, play, and sleep may stand in the way of being prepared for healthy sleep. An example is the expectation that workers are available 24/7, with work bleeding into leisure hours and reducing the time available for sleep [14] [9]. Another norm is around screen and device use, particularly phones; in a recent survey, $90 \%$ of Americans regularly used electronics before bed [8]. Having a mobile device in bed isn't always the individual's choice; for couples, it may be a partner using a smartphone or tablet in bed [51].

Finally, people may have misconceptions about sleep which prevent taking corrective measures accordingly. For instance, some people have a misconception that snoring does not interfere with sleep; still others believe insomnia strictly means difficulty falling asleep and therefore do not consider difficulty staying asleep as a problem needing to be addressed [50].

\section{Existing Technologies for Healthy Sleep}

Game design for health intervention with pervasive technology has gained much popularity, as we grow increasingly attached to our mobile phones. Examples include Time to Eat and OrderUP! [46] [24]. Specifically on interventions for healthy sleep, we find four major approaches in the literature: explicit instruction, sleep analytics, timing support, and reflection support. We note that some interventions designed to affect other outcomes (e.g. mindfulness) can also affect sleep.

Explicit instruction. In this approach, technologies educate users about factors related to sleep, from their environment to their routine to their daytime activity. Perfect Bedroom [39], for instance, offers information in the context of an edu- cational game. SleepCoacher gives individuals personalized recommendations [17].

Sleep analytics. A sleep analytic approach leverages the capacity of sensors (often on mobile phones) to categorize behaviors into asleep/awake with relatively high accuracy [40]. These data are then used to generate a visual model of sleep quality and duration, reflecting synthesized information back to the user of the phone. For example, ShutEye provides ambient feedback to users on their phone screen background about sleep-relevant activities [5]. iSleep uses the built-in microphone of the smartphone to detect the events that are closely related to sleep quality [26]. Sleep data is also a key facet of many lifelogging applications [35]. The analytics approach can expand beyond logging sleep activities to monitoring and capturing the sleep environment to help users understand the possible contributing factors to their sleep experience. Lullaby [37] utilizes a suite of sensors to provide a comprehensive recording of bedroom environment such as light, temperature and noise.

Timing support. One of the key contributing factors for healthy sleep is timing, which includes both regularity of schedules and the appropriate choice of sleep or wake time. iPhone Bedtime [33] helps users create and maintain a consistent sleep schedule. Similar applications strive to consolidate users' sleep schedule with their natural Circadian rhythm, such as the Philips Wake-up Light [1]. The EZwakeup sleep environment wakes people up slowly and smoothly from deep sleep [31].

Reflection support. Technology can help users be more aware of their decision-making around sleep. For example, instead of receiving generic sleep recommendations, users can receive personalized, cohort-based recommendations to support changing behavior [16]. The SleepBot project and SleepTight use text input to help users reflect on sleep decisions [49][13].

Implicit support. In addition to methods that explicitly seek to support healthy sleep, sleep can also be affected through mediating factors. For example, mindfulness has been shown to improve sleep quality [7], suggesting that mindfulness technologies indirectly promote healthy sleep indirectly. For example, emWave guides the user in stress reduction through excercises in patterned breathing [7].

\section{Game Design Approaches}

Previous work on sleep and games within HCI is limited. Existing approaches either seek to explicitly educate users [39], or to gamify sleep by adding rewards for sleep quantity or quality $[19,12]$. These approaches are relatively shallow uses of games as a medium [44]. We see opportunities for games to contribute much more to healthy sleep. We outline some of these opportunities in this paper.

The Transformational Game Framework [15] demonstrates the wide capacity of games to transform players, from changing attitudes to behaviors to social relationships. However, it emphasizes that in order to do so, there must be deep connections built between the problem and game design practice. These deep connections are typically understood to be best practices for game design [34]. The framework facilitates the development of these deep connections by providing a common 
language for game designers and researchers, and by providing exploratory questions that prompt the game development team to consider a range of features of the transformation.

While games can explicitly address sleep, the Embedded Design approach argues that subtle approaches to transformation can also powerfully impact players [36]. In some cases, embedded interventions in games can outperform more explicit approaches. This design strategy provides support for the notion that games do not have to have "face validity" in appearing to be about sleep in order to affect sleep.

Finally, games can create a wide range of player experiences or "aesthetics" [32]. For example, "cozy games" incorporate safety, ambience, and abundance while eschewing time pressure or individual responsibility [30]. Drawing on a wide range of aesthetic experiences in games can afford new approaches for sleepy game design.

\section{METHODS}

We used multiple methods to understand the design challenges of sleepy games. First, we explored the space through an iterative design process. We worked with sleep experts to define appropriate challenges, explored relevant literature, created sleepy game prototypes, and iterated them based on internal playtesting. Next, we analyzed the games as artifacts to draw out themes, and used this analysis to identify three games for external playtesting. Games were selected based on coverage of the themes. Finally, we playtested the three selected games with external participants and collected quantitative and qualitative data on their play experiences and their sleep.

\section{Game Design and Development}

Using a research through design approach [57], nine game design teams created sleepy game prototypes over the course of eight months. Many of the prototypes are comprised of both physical and digital components. Each interdisciplinary team was comprised of 3 - 4 students, ranging from undergraduate to $\mathrm{PhD}$ students, with backgrounds including art, computer science, design, psychology, human-computer interaction, educational technology and applied learning sciences. All students had at least some game design training and teams spent between 4 - 14 weeks developing their games.

In consultation with sleep experts, each team selected a user group that had known barriers to healthy sleep. Expert input was drawn from an industry partner; the range of input included interaction design, people research, medical product development, and sleep and respiratory care. Barriers to healthy sleep among the user groups included factors such as household responsibilities, physical pain, mood, scheduling, breathing, and environment. Teams conducted literature reviews and interviews with stakeholders to develop an understanding of the problem space and produce a transformational framework for their design [15]. Stakeholder types included intervention targets (person with sleep barrier), active players (people playing the game), and non-playing stakeholders (non-playing individuals affected by target's behavior). Teams moved from rough game sketches to physical prototypes. For internal playtesting, design teams exchanged playtests and critique, using the EOTA method to elicit high-quality feedback
[25]. Teams iterated their games a minimum of five times based on the feedback received.

\section{Design Analysis}

During the design process, teams produced a range of materials including designers' descriptions of the problem space, documentation of their design process, playtest notes, and multiple iterations of each game. This material was analyzed using methods drawn from Design Challenges for Livestreamed Audience Participation Games [23], to bring out common design challenges faced by different groups. Representatives from each team attended design meetings throughout the game design process. In addition to playtesting and providing peer feedback, these meetings provided an opportunity for designers to validate that the challenges described were ones they had faced in their process. To confirm their self-reports, a subset of the research team analyzed the written process data produced by each team. Teams used a grounded theory process as a way of making sense of the collected data [42]. Codes were developed for each of the design challenges described below, and two team members coded the process documentation which included combing the following materials: theory papers, process books, presentation slides, and (when applicable) game component documents. When the coders disagreed, they discussed the differences until they reached agreement.

Based on our coding of the process material, we were able to identify "coverage" of the design challenges across the different games. The challenges manifested differently for each game, due to differences in problem statement, game genre, materials, and other design choices. Since a full description of nine games is beyond the scope of this paper, we selected three games that together allowed us to explore the complete set of challenges. The selected games (Dream On, Good Night, and Office Shots) are described in detail in the design section of this paper; brief summaries of the other six games can be found in Appendix A.

\section{External Playtesting}

We conducted 12 external playtest sessions. In each, game materials were provided to players to play independently in an authentic context, and playtesters were provided with up to two full days to complete play. Dream On $(\mathrm{N}=6)$ was played by individuals while sleeping in an unfamiliar context, such as an Airbnb or new apartment. Good Night $(\mathrm{N}=4)$ had to be played in a multi-person household in which at least one member of the household maintained a sleep-wake schedule mismatched with others in the household. Office Shots $(\mathrm{N}=2)$ required a workplace scenario for workers to experience and interact with it as part of a daily routine.

We recruited participants who reported a range of sleep issues. Recruitment took place through flyers, email lists, and social media. Participants were eligible for the playtest if they experienced one of the relevant problems with sleep. Researchers assigned them to games based on the issues they reported, such that each participant played a game for which they were the intended audience (e.g. roommates with very different schedules playing Good Night). Playtesters ranged in age from 24 to 62 with a mean of 31.5. For multiplayer games (Good Night and 


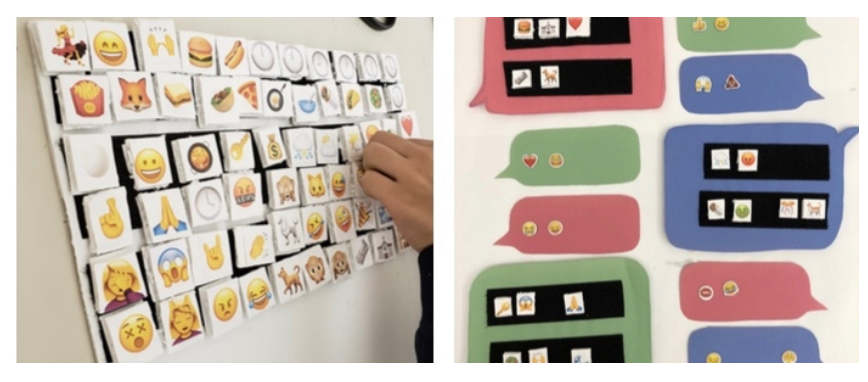

Figure 1: Good Night prototype

Office Shots), participants recruited a total of 14 co-players from the relevant environment (home or workplace). However, data was collected only from the participants themselves.

All games involved an initial preparatory meeting to introduce the game and hand it off to the participant. Participants had the opportunity to ask questions about the project and about gameplay. Participants also received written instructions for the game via email. During the preparatory meeting, participants were interviewed about experiences, challenges, values and priorities related to sleep (see Appendix B for questions). Pre-playtest interviews ranged in length from 12:52 to 29:32 with a mean of 19:44. After playtesting, participants were interviewed a second time. Post-game interviews included questions on experience and insights into playing the game along with the ways in which gameplay affected sleep habits, sleep quality, sense of control, and relationships with others (see Appendix C for questions). Post-game interviews ranged in length from 13:31 to 29:19 with an mean of 20:35.

Interviews were transcribed for analysis, using the codebook developed for the design analysis above. Two raters coded quotes and reached $92 \%$ agreement. Additionally, raters identified quotes that were surprising but did not fit into any of the existing codes. These elements were discussed as a team and used to augment our understanding of the player experience.

\section{GAME DESIGN}

In this section, we describe the design of our selected games (Dream On, Good Night, and Office Shots). Because design examples require context, we draw from these three games to illustrate our design challenges, and show how the experience carried through to external playtests. However, we provide a summary of our other six games in Appendix A, so that readers can understand the full scope of our designs. Additionally, the game Lights Out is described more fully in [47].

\section{Dream On}

Dream On responds to the challenge of sleep away from home. When sleeping in an unfamiliar place, the sleeper's brain maintains a partial state of arousal, leading to diminished quality of sleep [2]. To counteract this activation, Dream On seeks to induce a sense of calm in the player.

The game format is an 8.5" x 11" bound book, along with a box of scent tokens. It requires one player, and is meant to be played in bed, while in a strange place, before attempting to sleep. Players begin by opening the book to the first page to refer to the instructions. The book adopts the style of chooseyour-own-adventure books. Each page includes a brief story segment, along with two or more options for how the story might continue. Each option points to a different page in the book, and players randomly select and sniff a scent token to inspire their decision for which option to pick.

Each page of the book contains a large-format and beautifully shot photograph illustrating the action taking place in the story. Story segments are short and addressed to the reader in second person. The narrative focuses on simple, familiar scenes with friends, animals, and nature. These scenes are free of risk or threat, and appeal to the user's memories of happy times. Sensory descriptions evoke cozy objects and situations, such as warm cocoa, ambient nature sounds, or the texture of soft sheets [30]. The designers tested a digital book, but players described this as less desirable given the context of play (in bed) and commented that digital devices did not induce coziness.

Scents for the tokens come from essential oils. In addition to smelling the aromatherapeutic scents, taking a deep sniff helps players regulate their breathing and relax their chest prior to sleep. Also, using the scent tokens to guide player choice reduces the decision-making load on the player. They do not have to decide in advance the kind of story they will explore. They surrender some of that agency to their scent memory, drawing on submission as a pleasurable aesthetic of play [32].

\section{Good Night}

Good Night is a multi-player game to help people in the same household who have different sleep schedules maintain their social connection. The initiating or target player is understood as a household member who delays going to bed in order to connect with housemates. This pattern is common among night- or swing-shift workers with their families, but also occurs with college roommates and other groups who have differing schedules but strong social bonds.

The game is designed to be played in an ongoing, asynchronous way among all members of the household. All game elements can attach to the household's refrigerator with magnets. It is thereby physically placed in the heart of the house. Play materials include a) magnetic conversation bubbles surfaced with velcro, b) magnetic response bubbles, c) emoji with velcro backing, d) emoji with magnetic backing, and e) extra blank materials for player personalization (Fig. 1).

The game begins when the target player arrives home, typically while co-players are still sleeping. Using the emoji and text bubbles, they create an encoded message about their day on the refrigerator. When other household members awaken, they collectively or individually interpret the message and leave an emoji response in one of the response bubbles. This mechanism is adapted from texting; however, conversations on the physical board eliminate the immediacy in texting, so as to foster an environment where players must have their discussion in person. Each round of the game ends when all players gather and explain the messages they left for one another, often resulting in laughter and confusion as in the game Telephone. On the individual level, constructing a message 


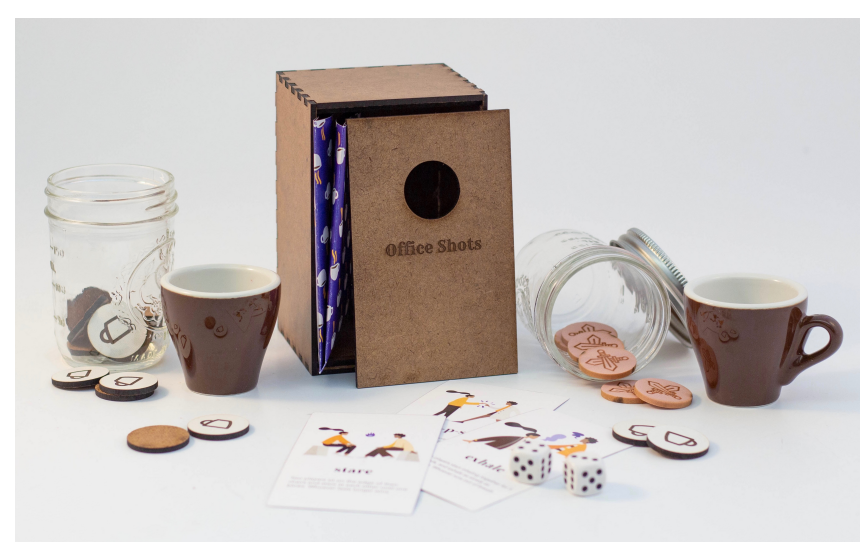

Figure 2: Office Shots prototype. Photograph by Judith Leng.

provides time for each player to reflect on their day, and to consider what is important for their family to know. At the same time, the playful ambiguity of the messages sparks conversation between players who are co-present in the space, since no player knows the meaning of all messages until the game ends. Finally, by playing, the household co-creates a physical artifact as a reminder of what they cherish in life.

\section{Office Shots}

Office Shots is a multiplayer social game, played in the workplace over a week. The goal is to help players be more intentional about caffeine consumption during the day, to improve sleep quality and timing at night. The game also seeks to change office culture around coffee, so that consuming large quantities in the name of productivity is not the default norm.

Game materials include coffee tokens, each representing the right to drink a cup of coffee; a token jar placed near the office coffee machine; a record sheet, which players keep with themselves to visualize their coffee consumption patterns; challenge tokens, which let players earn the right to more coffee; and a deck of mini-game cards (Fig. 2).

Each player receives six challenges tokens to start the game. Every morning, players meet at the coffee machine. Together, they decide how many cups of coffee they plan to collectively drink; the number of players is subtracted from their plan to create a coffee challenge number. That number of coffee tokens are issued to the group and split among the players.

After players disperse, gameplay continues pervasively during the workday. Each time a player drinks coffee, they must pay a token into the jar record the time and arousal level $(1-5)$ on sign their name on their record sheet. An earlier iteration used digital forms collected daily to track coffee consumption. However, they appeared to have helped designers understand players' behaviors, but did not lead to self-discovery for the players themselves. Therefore, the designers opted for the physical sheet, which players carry throughout. Since players are to record their arousal level each time they consume coffee, the sheet reinforces players' understanding of their own habits.
If players run out of coffee tokens, they may pay one of their challenge tokens to earn more coffee. To do so, they must challenge a co-worker to a mini-game. Mini-games are a key component of the game design, and are invoked when the player is craving coffee over and above their plan. Each mini-game asks the player to engage in activities that could temporarily substitute for caffeine as a stimulant, such as light physical exercise. If the challenging player wins, they receive an extra coffee token from the public jar and can use it to redeem coffee at any time before the end of the day; if they lose, they lose the said challenge token.

Another critical design element is built-in conversational dynamics. The morning token ritual encourages co-workers to talk about their coffee habits without losing face. There are no explicit rules governing how tokens must be shared, allowing players to negotiate distribution for themselves. Co-workers can offer one another tokens in acts of generosity, or set up informal token economies. Finally, the public location of the token jar and the act of engaging in mini-games invite conversation both between players and with non-playing co-workers.

The human element and the physical components are indispensable in facilitating players' externalization and reflection of their coffee consumption patterns. However, we believe several digital elements may streamline the game experience. For example, a system that digitizes the tokens and mini-game cards can prevent material loss and visualize personal coffee consumption trends over a longer period of time. As the game scales, the system can also learn from players' current coffee consumption and designate the total coffee quota for the players' next round of gameplay.

\section{DESIGN CHALLENGES AND PLAYTEST INSIGHTS}

In prior work, we listed seven key design challenges for sleepy games: agency and control, physiological and mental arousal, intervention timing, social embeddedness, multisensory experience, vulnerability, identity and values [47] (see Appendix D for a summary). In this paper, we expand our analysis in three ways. First, we draw examples of the design decisions that led us to the challenges from our games. Second, we provide playtest data related to the theme. Finally, when relevant, we share design tools that helped us address the challenge.

\section{Agency and Control}

Sleeping on demand is difficult, particularly for those who already struggle with healthy sleep. Moreover, the sense of not being in control of one's sleep can actually make it harder to sleep. Games, on the other hand, can be understood as "the art form of agency," which stretch our aesthetic experience of agency by providing finely tuned challenges [43]. A sleepy games approach to resolving this contradiction avoids asking players directly to try to sleep. Instead, the game provides a challenge within which players can experience agency; in turn, secondary effects of the challenge completion affect sleep.

In Dream On, the player is not directly asked to affect their sleep. Instead, they are given a task that they can easily accomplish: choosing their 'adventure' storyline. If players want to control their story's progress, they can flip ahead to preview each storyline; however, the stories are deliberately 

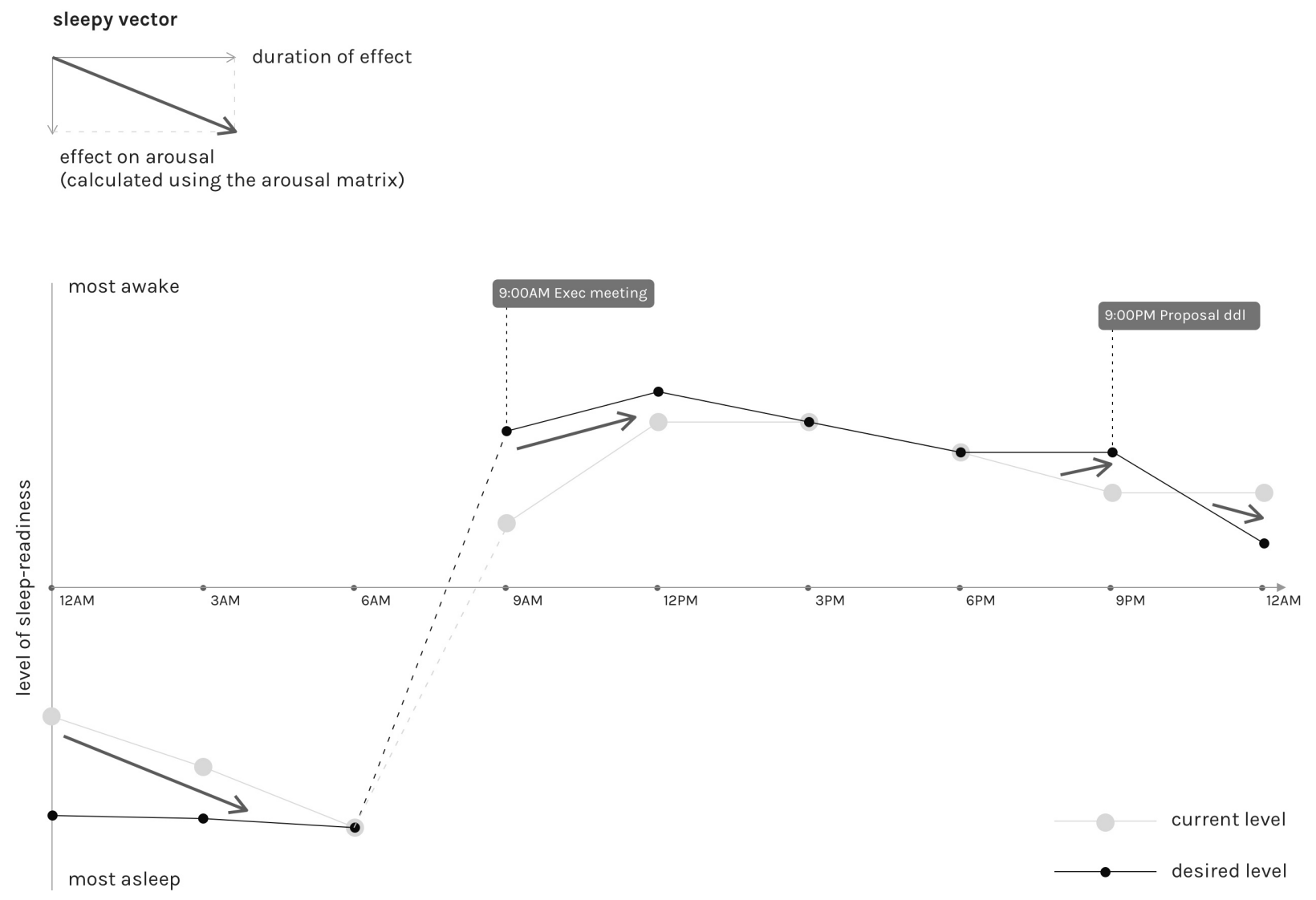

Figure 3: Priorities Timeline

undramatic, focusing instead on the experiential qualities of the narrative. Do you prefer the forest to the beach? Then your story centers on a peaceful hike. To amplify this effect, players are given an alibi for their choices. They do not need to attempt to seek out a particular ending or even to express their personal preferences, only smell one of the aromatherapy tablets and choose based on the memory it evokes. Players can complete the task without trying to control the task.

Players agreed that Dream On gave them a sense of control, not just within the game but over an environment that was not necessarily welcoming. P1 pointed out that "if you move into a new place, a new house, or if you have to sleep in airport,... you can control like your visuals, like lights and settings by Blackboard and stuff, right. Or audio through your phones, but you can't control, like, smell." While some settings (e.g. hotels) offer aromatherapy, P1 further argued that continuity of the scentscape across contexts was important. "If I had to actually move into a new place and then I already had this routine, um, I would just put this up on my nose and try to go to sleep and then maybe feel better."

\section{Physiological and Mental Arousal}

Racing hearts and racing minds can both make it difficult to sleep. This phenomenon is known as high physiological or mental arousal. However, arousal levels do not change instantly. For example, exercising (high arousal) early in the day can improve sleep (low arousal) later. The sleepy game approach recognizes that players' arousal levels may vary at the time of play, and that the desired level of arousal may vary depending on when play occurs. As we explored arousal levels, we realized that physiological and mental arousal did not always go hand in hand. We therefore needed ways to track the two separately. The arousal matrix (Fig. 4) helped us analyze arousal occurring within the gameplay. Physiological arousal refers to the functions of the body such as a change in breathing or heart rate, while mental arousal refers to cognitive processes. Mapping the game in this way helps to identify whether the game increases or decreases arousal overall, along with isolating potentially overstimulating features. From the visualization, the designer can determine when in the player's sleep-wake cycle the game might best be played.

Both Dream On and Office Shots directly manipulate players' physiological arousal. Dream On, which is played just before bed, seeks to lower players' arousal levels through aromatherapy. Players indeed described the experience of reading while smelling the aromatherapy tokens as "calming" (P6). Office Shots, on the other hand, manipulates arousal levels during the workday, when most people are not attempting to sleep. The game is targeted to players who already use caffeine as a stimulant to manage their arousal levels during the day. We found that many prospective playtesters were hesitant to participate because they were worried that limiting their access 


\begin{tabular}{|l|l|l|l|}
\hline \multicolumn{1}{|c|}{ game } \\
$\begin{array}{l}\text { arousal } \\
\text { level }\end{array}$ & $\begin{array}{l}\text { element 1 (e.g. } \\
\text { choose-your-own- } \\
\text { adventure storyline) }\end{array}$ & $\begin{array}{l}\text { element 2 (e.g. } \\
\text { soothing scents) }\end{array}$ & $\begin{array}{l}\text { element 3 (e.g. large, } \\
\text { sweeping images) }\end{array}$ \\
\hline mental arousal & + & - & -- \\
\hline $\begin{array}{l}\text { physiological } \\
\text { arousal }\end{array}$ & N/A & -- & -- \\
\hline
\end{tabular}

Figure 4: Arousal Matrix indicates whether game features increase (+) or decrease (-) mental and physiological arousal.

to caffeine would prevent them from handling their responsibilities. While targeting caffeine may have felt risky to these potential players, we see this as evidence that people actively manage their arousal levels during the day, and that games can provide new tools for doing so.

\section{Intervention Timing}

As suggested by the example of arousal levels, sleep is often mediated by behaviors that happen at non-sleeping times of the day. Additionally, sleep can be affected by long-term changes such as seasonal affective disorder. Sleepy games, therefore, do not necessarily need to be played at the moment of sleep. The sleepy game approach can incorporate gameplay at many times of day and on many timescales.

In order to design games in this extended temporal context, we needed to visualize the timeline of play. To do this, we created a tool we called the priorities timeline. First, the designers create a timeline of the player's day, labeled with routine events and timestamps. In particular, the designers capture the sleep-wake cycle of the target player as well as those of other participants in the game; events are mapped against arousal level. Second, the timeline is overlaid with other information about the player, such as their priorities or desires (see Fig. 3, presented using sample data for clarity and participant privacy). Last, designers' proposed game interactions are added to the map. If the game extends beyond a single day, the map can be repeated for the target length of playtime.

This tool proved critical for design in three ways. First, it allowed teams to be clear about when game elements might be deployed. Instead of saying "in the morning," they could locate gameplay relative to events of salience to the player. Designers could iterate their proposed game, and keep teammates on the same page, simply by moving events on the priorities timeline. Second, teams could identify opportunities to add gameplay elements. For example, if the game targeted the player at times of low mental arousal, inspecting the map could show designers when the player was in an appropriate state but no game activities were relevant. Finally, the priorities timeline was especially important for the design of multiplayer games, allowing the designers to better understand the impact of the same game on different stakeholders.

Both Good Night and Office Shots relied on timeline mapping to design player behaviors. In Good Night, different players from within the same household are interacting with the game at different times of day. Players interact with the game at the same relative time based on their sleep-wake cycle, but at different absolute times according to the clock. Office Shots alternates between strict and fluid temporal activities. For example, every work day begins with a kick-off for players to allocate their coffee tokens for the day. During the rest of the workday, players can choose to initiate additional challenges to gain more coffee tokens if they need an energy boost.

\section{Social Embeddedness}

Decisions about sleep are embedded in a social, rather than solitary, context. While no one else can sleep on your behalf, opportunities for healthy sleep are constrained by social factors. Players may share space with others, have caregiving responsibilities that interfere with sleep, or experience social pressures that affect their sleep choices. Sleepy games, therefore, consider the social space as design material. Sleepy games may ask the player to play within their social context by inviting others in their life to join, or to play with their social context by setting up temporary norms around sleep within the frame of the game.

Good Night helps amend the relationships between target players and household members who are on different schedules. The boards and conversation bubbles make room for asynchronous communication, open interpretation and the "overlap time" is an opportunity for players to engage in social interactions. Blank magnets further support the personalization and intimacy of the interaction. This customization allows players to draw their own uniquely relevant emojis and change them over time.

We found that participants were clearly aware of the ways in which their sleep decisions affected, and were affected by, others in the household. P5, for example, reported "if my [three and a half year old] brother is still kind of running around, it's um, one we need to go to sleep so that we can all go to sleep and get a reasonable amount of sleep, but also just to not wake up or wake him up or keep him up." Playtesters saw gameplay an opportunity to intervene in the social structure of the home: "I feel if I map this game directly to my life, I would in general maybe have a half an hour discussion everyday with my roommates where we openly discuss about things that we maybe shy about or feel inconvenient or awkward about then"(P13). Another playtester expressed the novelty of gaining insight into housemates, "It was interesting to see just what they did-the other two people" (P2).

\section{Multisensory Experience}

Designing for sleep means designing for all five senses. Sleepy games consider not only players' mental and emotional engagement with play, but also their embodied experience of the game. A sleepy game design process treats the aesthetics of game objects as critical; this design concern may also manifest in the environment in which the game is played.

In Dream On, this design choice manifests both in what is included in the game, and what is excluded. The design of the game's physical materials evokes strong cues of relaxation. In addition to the game's scents, each page of the game book features a large, beautiful photograph. The book itself is small enough to be comfortably held while in bed, but large enough to allow the photographs to have a substantial visual 


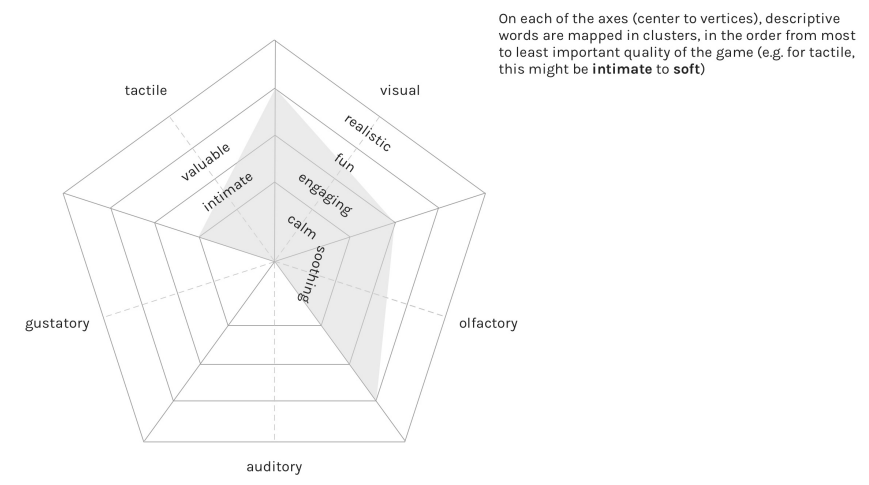

Figure 5: Multisensory Spider Word Cloud

impact. On the other hand, the game book could have been provided in a digital rather than a physical format. Choosing a physical presentation, and omitting screens, kept the player from establishing screens as part of this relaxing ritual.

We used a multisensory spider word cloud to align the desired sensory experience of the game with playtest results, which helped both with game iteration and interpreting player response (see Fig. 5). Keywords were pulled from two sources. The first is mapping descriptive sensory words based on the designers' aims. The second is sample game descriptions as provided by playtesters when describing any of the following: 1) physical, physiological, and mental state during/after playing the game, 2) aesthetics of the physical features of the game, or 3) other sensory descriptions. By comparing and overlapping the spider maps, the designer can see where their designs are as expected and where there are surprises.

Playtesters were aware of the role of sensory factors in sleep. $\mathrm{P} 2$ pointed out that they make it hard for their roommate to sleep because "I'm noisier than they would like" and "use a lot of lights cause it helps me wake up, so they probably get exposed to a little bit more light than they would like." Players described their sensory experiences in the games not only as pleasing on the sensory interaction level, but as affecting their overall experience: "Having the smells available made it easier to relax and actually just focus on the game itself...having that extra sensory bit made it easier to concentrate and forget about all the other things that usually swirl around my mind" (P3).

\section{Vulnerability}

Sleep is a vulnerable state. Sleepers cannot directly perceive what is around them (although in unfamiliar environments, people maintain partial arousal and do not sleep well [2]), nor can they deliberately act to control their environment. Sleepy games use play to address players' sense of vulnerability around sleep. The sleepy game approach is willing to tackle sensitive or intimate topics, using embedded design techniques and the psychological distancing effect of play to help players embrace their vulnerability and transform behaviors that might otherwise be protected by player defensiveness. Examples of pre-play Dream On interviews convey how participants feel about sleeping in an unfamiliar place prior to game play. P8 stated, "I like to kind of manage the space that I'm sleeping in and put it to my liking at least before I go to bed. If possible, I usually bring my own stuff with me. So I would bring a blanket up, bring a pillow and sometimes my own sheets. So I try to keep the things mine and I'm familiar with them as much as possible". The sense of vulnerability that comes from unfamiliarity is described by one participant as "the house that I'm subletting-I guess other people do have keys to it-but it's just me there right now. So that's kind of creepy. It's like this huge house. Oh my God, it's the worst at night" (P3).

Playtesting suggested that games can mitigate the discomfort that comes with sleeping in an unfamiliar place. One participant became so comfortable during playtesting that they chose to play the game unclothed. "I'm the only person in...the Airbnb home...so because of that, I took the opportunity to do something that I don't really ever have the chance to do. And that was, I played the game and did other things almost naked in the place...I do have body image issues and so just the fact that I could be in an almost completely physically vulnerable state, but like doing whatever, it just felt nice" (P5).

\section{Identity and Values}

Sleep decisions are intertwined with sleepers' identities and values. For example, people may see staying up late to work as a signal that they are industrious and committed, even if their late-night work impairs their overall productivity. (One of our playtesters reported precisely this dynamic: "I watched Youtube video for 2 hours and then I worked and then it's 2:00 AM I'll just feel good about myself because it's 2:00 AM and I worked till 2:00" (P1). Sleepy Games honor players' values around sleep, but can also contribute to shaping them.

Office Shots was designed to help players reflect on their relationship with caffeine, and the broader social context of which coffee is a marker. Regulating one's ability to work using caffeine is seen as a requirement in high-pressure workplaces: "a lot of my immediate peers and colleagues were always talking about like working on the weekends or working late at night and all of this pressure and I just really don't like that. And it came to it where everyone was talking about it so much that like either I stopped hanging out with people who would only talk about that, or I said like: Hey, if we're hanging out socially, I would prefer you don't talk about work" (P12).

To accomplish this goal, the game components of Office Shots are physically located in a communal space near the coffee machine. This design decision establishes a new stake in the ground over the coffeepot, diffusing the default baseline of the coffee machine as a symbol of productivity and overwork and re-imagining it as an opportunity for workers to act on a new or preferred set of values. Even non-players noticed the game, since it was in common space, and used it to foster discussion: "[a co-worker] was asking what the challenges were. So it was like a talking point for all of us that was not work related...it was a positive thing in the culture" (P7).

\section{PLAYER IMPACT}

In playtesting, we sought to understand and validate our design challenges. We did not explicitly measure behavior change, as the games are meant to serve as ongoing interventions 
in players' lives. We nonetheless observed that even in the relatively short playtests, games affected players as follows.

Players were highly engaged by the games, and enthusiastic about exploring new solutions to increase sleep quality. For example, playtesters were not explicitly instructed to play the given game more than once; however, 11 of 12 playtesters voluntarily played their given games for the entire two-day period. This included both players who had a prior history of experimenting to improve sleep (P1 had tried lucid dreaming, P8 avoids coffee and alcohol, P11 changed the timing of their meals) and players who had not previously attempted interventions. Only 3 of 12 participants reported feeling in control of their sleep habits, which may have motivated their engagement with a novel type of intervention. However, players engaged beyond simply playing the games; they used the games as platforms for sleep experimentation. For example, P1 subjected himself to different types of media stimuli before trying Dream On, while P3 varied the times at which she played the game, and P5 experimented with playing while nude.

Across all three games, players reported increased awareness of their sleep-related choices. P12 better understood their "patterns of behavior" around coffee: "if I skipped breakfast in the morning at home, I drink coffee at work cause I'll go to the cafe and pick up like a pastry and a coffee. I won't just pick up the pastry." P1 described how they adapted their pre-bed routine to incorporate Dream On: "normally I would just do nothing, just play videos, like watch video til I go to sleep, right. But now... I will pause the video and then do this, then maybe resume watching it or like just go to bed right after." Good Night increased players' social awareness. While playing, "you're not completely in your own head all the time" (P2) and become aware of the needs of others in the household. Players also became more aware of their values around sleep. For example, P3 talked about sleep habits in her family of origin; P1, as described above, sacrificed sleep to feel industrious; P12 reflected on the impossible performance expectations for graduate students through the lens of coffee.

For the multiplayer playtests $(\mathrm{N}=6)$, participants also reported having positive and unexpected social interactions. Five of six players felt they understood their fellow players better. Some (e.g. P12) said they learned specifically about aspects related to sleep, such as coffee consumption. Others simply reported increased closeness. P9, for example, reported that family members were very interested in the game, his behavior, and his involvement in research. Office Shots became a talking point in P7's office, both with participating players and nonparticipating co-workers. P9 reported engaging with his son around Good Night thanks to a social media post from his participating daughter. Many conversations occurred outside the play activity itself. For example, P12 texted with a coworker about Office Shots outside of work hours.

Short-term measures are not always the most effective way to change sleep habits; for example, P3 reported "when I first start trying out the sleep routine, I'm able to do it for like two or three days. But then after that I can't stick to it." However, impacts of even these short periods of play suggest opportunities for longer-term study.

\section{DISCUSSION}

\section{Sleepy games as a creative practice}

In external playtests, we observed that participants produced artifacts related to sleep, over and above any artifacts produced by gameplay or by data collection. Of our 12 participants, 7 engaged in this behavior unprompted. The most common forms of artifact creation were sleep logs, photographs of gameplay, and diary-based reflections on sleep. Some participants also reported that other people in their life created artifacts around gameplay. For example, P9 described interacting with both his son and daughter around spontaneously created artifacts. "My daughter, you know, Snapchated or Instagram ... put it on social media. And my son saw that and he had it. "What are you doing, Dad?" So even though he wasn't part of it, he doesn't live with us. My daughter shared it to the world, with the world, yeah, out there."

Many games, such as Minecraft [41], allow user-generated content. It is possible, therefore, that the production of these artifacts is partly inspired by using the language of games to engage players with sleep. Sleep is not something that sleepers necessarily know how to achieve, let alone creatively contribute to. However, games provide structured artifacts and interactions that allow players to be creative. We hypothesize, therefore, that we can use these artifacts to redirect player agency into a sense of agency around sleep activities.

Playful creativity may provide a new approach to sensemaking with health data, suggesting relevance to the wider HCI community as health data is increasingly collected. As smartphone sensing matures [38] and both automated and manual health data collection increases [11], the design space that is the boundary between expected, sensed, and desired sensor data [6] is expanded. Existing work shows that interactions with sleep data can be counterproductive for healthy sleep, because players feel the data is asking them to accomplish the impossible [48]. Our artifacts take a different approach. Instead of using the data to try to affect sleep, players were creating what we understand as "souvenirs" of sleep based on game experiences. Artifacts were used for reflection, meaningmaking, and social connection. Participants reported a sense of "calm" (P5), "excitement" (P2) and feeling "more...positive" (P6) about their artifacts, which matches the recommendations of [48]. In future, we could explore designing for souvenirs directly, drawing on games such as Threadsteading [3].

Finally, we note that production of sleep souvenirs introduces privacy issues. One might imagine that instead of sharing photographs with her brother, P9's daughter could have shared them with someone P9 did not want to see them. Ownership of sleepy game artifacts is likely to be negotiated socially, so technical systems must support a range of sharing options.

\section{Sleepy games as a social practice}

In our design work to date, we focused on designing games that are played by the person with the sleep issue. However, our findings demonstrate that this is actually an assumption. Sleep is a social practice; the person playing a sleepy game is both affecting, and being affected by, other people in their life. This interpersonal affect could occur within the home, as in 
the case of families with young children [10], or in work or social contexts outside the home. For instance, P12 describes Office Shots prompting "interesting conversations" with an office mate when accessing the coffee tracking sheet.

We can use this insight to design games for people adjacent to a sleep problem. The direct stakeholder in a sleep problem may not always be the one seeking help. Consider a person who is convinced that they can function on too-little sleep. They are unlikely to initiate change. One could appeal to them with a game that does not explicitly address sleep, using an embedded design approach [36]. However, considering sleep behavior change as a social externality of play suggests another approach to the design. The player might be indirectly affected by the sleep problem, while the game affects the person directly affected. This approach is supported by prior work; sleep interventions for families with small children, for example, must address the family system [10, 47].

This approach also suggests ways to inform timing support interventions. Instead of considering sleep/wake timing as an absolute, users may value sleep/wake timing relative to their social context. For example, designs can leverage the value that sleepers put on their relationships by showing how changes in their sleep habits can give them more time with the people they love. Data from multiple parties would therefore be needed to produce relational recommendations.

\section{Sleepy games as a situated practice}

In our games, we observed that players were willing to modify their environment as part of gameplay. For example, Office Shots involved adding game materials to a shared office space; Good Night took up real estate on the family fridge; and even Dream On required players to make space for it in their luggage while traveling. Some of this willingness may have been due to novelty, or to users' sense of social obligation to playtest the game. However, we see this as an opportunity to use choices made during gameplay to affect the physical or social situation in which sleep takes place.

We observed that many players were already experimenting with their sleep habits; though, they also reported finding it challenging to sustain changed behavior. Making changes playful can help players try new things, regardless of their current willingness to experiment. For example, P5 tried sleeping in the nude although it was a challenge for them to do so. Playfulness can also help players view their physical and social environment with new eyes, rather than becoming creatively locked in to their current interpretations of objects and spaces [56]. This approach can affect how reflection on sleep practices works even outside the game context. Instead of reflecting through talking or looking at data, players can reflect through playfully modifying their environment, such that sleepers become designers of their own experience, probing their own life to better understand the impact of their choices.

\section{LIMITATIONS}

Our study was not intended to be comprehensive of all possible approaches to sleepy games. While we believe we provide a useful framework, we look forward to expanding it in the future. In particular, we emphasized non-digital prototyping and playtesting. We expect to see new designs emerge from "born-digital" sleepy games. Could a sleepy game be 'played' while sleeping by, for example, connecting with data from on-body or bed-based sensors? Could co-sleepers compete or cooperate? We anticipate this study will activate further and future questions about the limits of sleepy games.

In our playtests, we recognize that we worked with a limited number of participants in only one American city, and that we selected only three of our games for external playtests. Additionally, we focused only on a narrow range of sleep problems derived from the design goals of those three games. Our playtest period was short, which means that players may have still been focused on the novelty of the game rather than its quality. Finally, we did not collect data on sleep outcomes, only on player behavior and experiences. We will continue our work in this area to address these issues.

\section{CONCLUSION AND FUTURE WORK}

In this paper, we present a design approach to healthy sleep that we call "sleepy games." Over eight months, we iteratively prototyped nine sleepy games and conducted external playtests with three of them. Through analysis of our design artifacts and playtest documentation, we identified seven overarching challenges of designing games for sleep (agency and control; physiological and mental arousal; intervention timing; social embeddedness; multisensory experience; vulnerability; and identity and values) and understand sleep as a creative, social, and situated practice. These games are not (yet) meant to improve sleep; rather, to engage players in sleep-relevant ways that build on proven theories and interventions. Evaluation of actual sleep improvements across varied lengths of time is part of our future work, just as Grimes et al tracked the effects of OrderUP! on player eating habits over a 3-week period [24].

In addition to continuing to explore the implications of our seven challenges, we will also explore the novel design directions from our discussion, such as designing for sleep souvenirs. We will collaborate with other sleep researchers to incorporate our findings into non-game interventions, and we will conduct evaluative studies to determine the impact of game and non-game interventions created with these principles. We look forward to many hours of happy sleep.

\section{ACKNOWLEDGEMENTS}

We thank our fellow designers: Jeffrey Chou, Tithi Jasani, Adela Kapuscinska, Ruoxi Li, Arnav Mahajan, Emily McDonald, Stacie Nam, Khushi Shah, Jessica Timczyk and Rocky Wang from Sleepy Games independent study in 2019; as well as the designers from the Transformational Game Design Studio class in 2018. We are grateful to Peter Weeks, Stephan Morgan, and Tom Bonnell of Philips Health, both for sharing their valuable expertise on sleep and for their generous support of this work. Finally, many thanks to all our interviewees and playtesters. We could not have made our games without you.

\section{REFERENCES}

[1] Philips Personal Health; a division of Philips North America LLC. 2019. Philips Wake-up Light. (2019). 
[2] HW Agnew Jr, Wilse B Webb, and Robert L Williams. 1966. The first night effect: an Eeg study of sleep. Psychophysiology 2, 3 (1966), 263-266.

[3] Lea Albaugh, April Grow, Chenxi Liu, James McCann, Gillian Smith, and Jennifer Mankoff. 2016. Threadsteading: Playful Interaction for Textile Fabrication Devices. In Proceedings of the 2016 CHI Conference Extended Abstracts on Human Factors in Computing Systems. ACM, 285-288.

[4] Bruce M Altevogt, Harvey R Colten, and others. 2006. Sleep disorders and sleep deprivation: an unmet public health problem. National Academies Press.

[5] Jared S Bauer, Sunny Consolvo, Benjamin Greenstein, Jonathan Schooler, Eric Wu, Nathaniel F Watson, and Julie Kientz. 2012. ShutEye: encouraging awareness of healthy sleep recommendations with a mobile, peripheral display. In Proceedings of the SIGCHI conference on Human factors in computing systems. ACM, 1401-1410.

[6] Steve Benford, Holger Schnädelbach, Boriana Koleva, Rob Anastasi, Chris Greenhalgh, Tom Rodden, Tom Rodden, Jonathan Green, Ahmed Ghali, Tony Pridmore, and others. 2005. Expected, sensed, and desired: A framework for designing sensing-based interaction. ACM Transactions on Computer-Human Interaction (TOCHI) 12, 1 (2005), 3-30.

[7] Karen Caldwell, Mandy Harrison, Marianne Adams, Rebecca H Quin, and Jeffrey Greeson. 2010. Developing mindfulness in college students through movement-based courses: effects on self-regulatory self-efficacy, mood, stress, and sleep quality. Journal of American College Health 58, 5 (2010), 433-442.

[8] Anne-Marie Chang, Daniel Aeschbach, Jeanne F Duffy, and Charles A Czeisler. 2015. Evening use of light-emitting eReaders negatively affects sleep, circadian timing, and next-morning alertness.

Proceedings of the National Academy of Sciences 112, 4 (2015), 1232-1237.

[9] Stella Chatzitheochari and Sara Arber. 2009. Lack of sleep, work and the long hours culture: evidence from the UK Time Use Survey. Work, Employment and Society 23, 1 (2009), 30-48.

[10] Anna Cherenshchykova and Andrew D Miller. 2019. Family-Based Sleep Technologies: Opportunities and Challenges. In Extended Abstracts of the 2019 CHI Conference on Human Factors in Computing Systems. ACM, LBW1718.

[11] Eun Kyoung Choe, Saeed Abdullah, Mashfiqui Rabbi, Edison Thomaz, Daniel A Epstein, Felicia Cordeiro, Matthew Kay, Gregory D Abowd, Tanzeem Choudhury, James Fogarty, and others. 2017. Semi-automated tracking: a balanced approach for self-monitoring applications. IEEE Pervasive Computing 16, 1 (2017), 74-84.
[12] Eun Kyoung Choe, Julie A Kientz, Sajanee Halko, Amanda Fonville, Dawn Sakaguchi, and Nathaniel F Watson. 2010. Opportunities for computing to support healthy sleep behavior. In CHI'10 Extended Abstracts on Human Factors in Computing Systems. ACM, 3661-3666.

[13] Eun Kyoung Choe, Bongshin Lee, Matthew Kay, Wanda Pratt, and Julie A Kientz. 2015. SleepTight: low-burden, self-monitoring technology for capturing and reflecting on sleep behaviors. In Proceedings of the 2015 ACM International Joint Conference on Pervasive and Ubiquitous Computing. ACM, 121-132.

[14] Marc Cowling. 2007. Still At Work? An empirical test of competing theories of long hours culture. (2007).

[15] Sabrina Culyba. 2018. The Transformational Framework. Unpublished manuscript (2018).

[16] Nediyana Daskalova, Bongshin Lee, Jeff Huang, Chester Ni, and Jessica Lundin. 2018. Investigating the effectiveness of cohort-based sleep recommendations. Proceedings of the ACM on Interactive, Mobile, Wearable and Ubiquitous Technologies 2, 3 (2018), 101.

[17] Nediyana Daskalova, Danaë Metaxa-Kakavouli, Adrienne Tran, Nicole Nugent, Julie Boergers, John McGeary, and Jeff Huang. 2016. SleepCoacher: A personalized automated self-experimentation system for sleep recommendations. In Proceedings of the 29th Annual Symposium on User Interface Software and Technology. ACM, 347-358.

[18] Danice K Eaton, Laura Kann, Steve Kinchen, Shari Shanklin, James Ross, Joseph Hawkins, William A Harris, Richard Lowry, Tim McManus, David Chyen, and others. 2008. Youth risk behavior surveillance-United States, 2007. Morbidity and mortality weekly report. Surveillance summaries (Washington, DC: 2002) 57, 4 (2008), 1-131.

[19] Kazuki Egashira, Yuki Furukawa, Yuuki Nishiyama, Tadashi Okoshi, Jin Nakazawa, and Hideyuki Tokuda. 2016. Poster: NiSleep: Gamification-friendly quantitative evaluation methodology of sleep quality. In Proceedings of the 14th Annual International Conference on Mobile Systems, Applications, and Services Companion. ACM, 132-132.

[20] Centers for Disease Control, Prevention (CDC, and others. 2012. Short sleep duration among workers-United States, 2010. MMWR. Morbidity and mortality weekly report 61, 16 (2012), 281.

[21] Russell G Foster and Leon Kreitzman. 2014. The rhythms of life: what your body clock means to you! Experimental physiology 99, 4 (2014), 599-606.

[22] Lisa Gallicchio and Bindu Kalesan. 2009. Sleep duration and mortality: a systematic review and meta-analysis. Journal of sleep research 18, 2 (2009), 148-158. 
[23] Seth Glickman, Nathan McKenzie, Joseph Seering, Rachel Moeller, and Jessica Hammer. 2018. Design Challenges for Livestreamed Audience Participation Games. In Proceedings of the 2018 Annual Symposium on Computer-Human Interaction in Play. ACM, 187-199.

[24] Andrea Grimes, Vasudhara Kantroo, and Rebecca E Grinter. 2010. Let's play!: mobile health games for adults. In Proceedings of the 12th ACM international conference on Ubiquitous computing. ACM, 241-250.

[25] Jessica Hammer and Amy Cook. 2018. EOTA: A Method for Improving Peer Feedback in the Game Design Classroom. In International Academic Conference on Meaningful Play 2018.

[26] Tian Hao, Guoliang Xing, and Gang Zhou. 2013. iSleep: unobtrusive sleep quality monitoring using smartphones. In Proceedings of the 11th ACM Conference on Embedded Networked Sensor Systems. ACM, 4.

[27] Peter Hauri. 1977. The sleep disorders. Upjohn.

[28] Shigekazu Higuchi, Yutaka Motohashi, Yang Liu, and Akira Maeda. 2005. Effects of playing a computer game using a bright display on presleep physiological variables, sleep latency, slow wave sleep and REM sleep. Journal of sleep research 14, 3 (2005), 267-273.

[29] Max Hirshkowitz, Kaitlyn Whiton, Steven M Albert, Cathy Alessi, Oliviero Bruni, Lydia DonCarlos, Nancy Hazen, John Herman, Paula J Adams Hillard, Eliot S Katz, and others. 2015. National Sleep Foundation's updated sleep duration recommendations. Sleep Health 1, 4 (2015), 233-243.

[30] Chelsea Howe, Daniel Cook, Jake Forbes, Dan Hurd, Tanya X Short, Squirrel Eiserloh, and Anthony Ordon. 2018. Cozy Games. Blog. (24 January 2018). Retrieved October 9, 2018 from

www. lostgarden. com/2018/01/cozy-games.html.

[31] Ming-Chun Huang, Xiaoyi Zhang, Wenyao Xu, Jason Liu, and Majid Sarrafzadeh. 2014. EZwakeup: A Sleep Environment Design for Sleep Quality Improvement. In CHI'14 Extended Abstracts on Human Factors in Computing Systems (CHI EA '14). ACM, New York, NY, USA, 2389-2394. DOI :

http://dx.doi.org/10.1145/2559206.2581250

[32] Robin Hunicke, Marc LeBlanc, and Robert Zubek. 2004. MDA: A formal approach to game design and game research. In Proceedings of the AAAI Workshop on Challenges in Game AI, Vol. 4. 1722.

[33] Apple Inc. 2019. Bedtime. (2019).

[34] Katherine Isbister, Mary Flanagan, and Chelsea Hash. 2010. Designing games for learning: insights from conversations with designers. In Proceedings of the SIGCHI Conference on Human Factors in Computing Systems. ACM, 2041-2044.

[35] Kasthuri Jayarajah, Meera Radhakrishnan, Steven Hoi, and Archan Misra. 2015. Candy Crushing Your Sleep. In
Adjunct Proceedings of the 2015 ACM International Joint Conference on Pervasive and Ubiquitous Computing and Proceedings of the 2015 ACM International Symposium on Wearable Computers (UbiComp/ISWC'15 Adjunct). ACM, New York, NY, USA, 753-762. DOI :

http://dx.doi.org/10.1145/2800835.2804393

[36] Geoff Kaufman and Mary Flanagan. 2015. A psychologically "embedded" approach to designing games for prosocial causes. Cyberpsychology: Journal of Psychosocial Research on Cyberspace 9, 3 (2015).

[37] Matthew Kay, Eun Kyoung Choe, Jesse Shepherd, Benjamin Greenstein, Nathaniel Watson, Sunny Consolvo, and Julie A Kientz. 2012. Lullaby: a capture $\&$ access system for understanding the sleep environment. In Proceedings of the 2012 ACM conference on ubiquitous computing. ACM, 226-234.

[38] Nicholas D Lane, Emiliano Miluzzo, Hong Lu, Daniel Peebles, Tanzeem Choudhury, and Andrew T Campbell. 2010. A survey of mobile phone sensing. IEEE Communications magazine 48, 9 (2010), 140-150.

[39] Maria Emanuela Matos Leonardo and Katie Moraes de Almondes. 2018. Study Protocol of Sleep Education Tool for children: serious game "Perfect Bedroom: learn to sleep well". Frontiers in psychology 9 (2018), 1016.

[40] Jun-Ki Min, Afsaneh Doryab, Jason Wiese, Shahriyar Amini, John Zimmerman, and Jason I Hong. 2014. Toss'n'turn: smartphone as sleep and sleep quality detector. In Proceedings of the SIGCHI conference on human factors in computing systems. ACM, 477-486.

[41] Mojang. 2011. Minecraft. Game [SNES]. (18 November 2011). Mojang, Stockholm, Sweden. Last played August 2011.

[42] Michael J Muller and Sandra Kogan. 2010. Grounded theory method in HCI and CSCW. Cambridge: IBM Center for Social Software (2010), 1-46.

[43] C Thi Nguyen. 2018. Games: Agency as Art. (2018).

[44] Scott Nicholson. 2015. A recipe for meaningful gamification. In Gamification in education and business. Springer, 1-20.

[45] Evy Öhrström, Emina Hadzibajramovic, Maria Holmes, and Helena Svensson. 2006. Effects of road traffic noise on sleep: Studies on children and adults. Journal of environmental psychology 26, 2 (2006), 116-126.

[46] John Pollak, Geri Gay, Sahara Byrne, Emily Wagner, Daniela Retelny, and Lee Humphreys. 2010. It's time to eat! Using mobile games to promote healthy eating. IEEE Pervasive Computing 9, 3 (2010), 21-27.

[47] Juliet Pusateri, Judith Leng, Jessica Timczyk, Xiangzhu Chen, Qian Wang, Khushi Shah, Tithi Jasani, and Jessica Hammer. 2019. Toward a Design Theory of Sleepy Games. In Extended Abstracts of the 2019 Annual Symposium on Computer-Human Interaction in Play. ACM. 
[48] Ruth Ravichandran, Sang-Wha Sien, Shwetak N Patel, Julie A Kientz, and Laura R Pina. 2017. Making sense of sleep sensors: How sleep sensing technologies support and undermine sleep health. In Proceedings of the 2017 CHI Conference on Human Factors in Computing Systems. ACM, 6864-6875.

[49] Steven R Rick, Aaron Paul Goldberg, and Nadir Weibel. 2019. SleepBot: encouraging sleep hygiene using an intelligent chatbot. In Proceedings of the 24th International Conference on Intelligent User Interfaces: Companion. ACM, 107-108.

[50] Rebecca Robbins, Michael A Grandner, Orfeu M Buxton, Lauren Hale, Daniel J Buysse, Kristen L Knutson, Sanjay R Patel, Wendy M Troxel, Shawn D Youngstedt, Charles A Czeisler, and others. 2019. Sleep myths: an expert-led study to identify false beliefs about sleep that impinge upon population sleep health practices. Sleep health (2019).

[51] Tarja Salmela, Ashley Colley, and Jonna Häkkilä. 2019. Together in Bed?: Couples' Mobile Technology Use in Bed. In Proceedings of the 2019 CHI Conference on Human Factors in Computing Systems (CHI'19). ACM, New York, NY, USA, Article 502, 12 pages. DOI : http://dx.doi.org/10.1145/3290605.3300732

[52] Venkatramanujam Srinivasan, Jarnail Singh, Seithikurippu R Pandi-Perumal, Gregory M Brown, David Warren Spence, and Daniel P Cardinali. 2010. Jet lag, circadian rhythm sleep disturbances, and depression: the role of melatonin and its analogs. Advances in therapy 27, 11 (2010), 796-813.

[53] Patricia Tassi, Mahnaz Saremi, Sarah Schimchowitsch, Arnaud Eschenlauer, Odile Rohmer, and Alain Muzet. 2010. Cardiovascular responses to railway noise during sleep in young and middle-aged adults. European journal of applied physiology 108, 4 (2010), 671-680.

[54] Matthew Walker. 2017. Why we sleep: Unlocking the power of sleep and dreams. Simon and Schuster.

[55] Matthew P Walker, Tiffany Brakefield, J Allan Hobson, and Robert Stickgold. 2003. Dissociable stages of human memory consolidation and reconsolidation. Nature 425, 6958 (2003), 616.

[56] Kristin Williams, Rajitha Pulivarthy, Scott E. Hudson, and Jessica Hammer. in press. Understanding Family Collaboration Around Lightweight Modification of Everyday Objects in the Home. In Proceedings of the 19th ACM Conference on Computer-Supported Cooperative Work \& Social Computing. ACM.

[57] John Zimmerman, Jodi Forlizzi, and Shelley Evenson. 2007. Research through design as a method for interaction design research in HCI. In Proceedings of the SIGCHI conference on Human factors in computing systems. ACM, 493-502. 